\title{
Meta-análisis de la influencia de diferentes indicadores reproductivos sobre la productividad numérica de las cerdas
}

\author{
Meta-analysis of the influence of different reproductive indicators of \\ numerical sows productivity \\ Yulien Fernández Romay ${ }^{1}$, Daniel Babot Gaspa ${ }^{2}$, Lluis Plà Aragónes², Yury Peña \\ RUEDA $^{3}$ y MARLENE BARBA RAMireZ ${ }^{1}$
}

\section{RESUMEN}

El presente trabajo pretende determinar, a través del meta-análisis, el nivel de importancia que tienen los principales factores que intervienen en la productividad numérica de las cerdas. Se seleccionaron 96 citas de diferentes referencias bibliográficas: bases de datos; revistas reconocidas internacionalmente con factor de impacto en Europa y América, publicaciones procedentes de Universidades de reconocimiento internacional y artículos publicados en eventos internacionales. Se establecieron criterios de inclusión y se codificaron los factores para su análisis estadístico. Los resultados permitieron determinar el grado y sentido de la influencia que tienen los factores que, a juicio de los autores, modifican la productividad numérica de las cerdas y demostraron que según las investigaciones realizadas los factores que mayor influencia tienen son la alimentación, prolificidad por orden de parto y duración de la lactación.

Palabras clave: productividad numérica; prolificidad; meta-análisis.

\begin{abstract}
Escuela Superior Politécnica de Chimborazo. Riobamba, Ecuador.

2 Universidad de Lleida. Lleida, España.

3 Universidad de Holguín «Oscar Lucero Moya». Holguín, Cuba.
\end{abstract}

This research aims to determine, through the meta-analysis, the level of importance of the main factors involved in the numerical productivity of sows. The 96 citations from different references were selected: databases; internationally recognized journals with impact factor in Europe and America, publications from internationally recognized Universities and articles published in international events. Inclusion criteria were established and factors for statistical analysis were coded. The results allowed to determine the extent and direction of the influence of the factors which, in the opinion of the authors, modify the numerical productivity of sows and showed that according 
to research the factors that most influence are food, prolificacy order childbirth and lactation length.

Keywords: numerical productivity; prolificacy; meta-analysis.

\section{ICHIKLLACHAW}

Kay urya musyapakuyqa meta-analisis nishqanwan tita aqtsa yupay mirayninpaqmi willakun. Tsaypaq 96 qillqashqa maytukunatam akrakashqa: willakuy kashqanta, hatun wak suyukunachaw ichik musyapakuy qillqashqata, kaykuna hatun yachay wayikunachaw riqitsikamushqanta, hinamanpis hatun willakuychaw yachaqkuna rimayanqantam. Tsaypaq istadistiku nishqan analisistam wanakashqa. Kay musyapakuyqa rikaatsimantsik musyaqkunapa qillqaynichaw imanawmi tita aqtsakunapa yupay miraynin tikranqantam, tsay musyapakuy rikaatsikun imanawmi mikuykuna awqanqantam, wachaypa miraynintawan chuchuychaw nakaynintam.

Pushaq shimikuna: yupaypa wachaynim; prolificidanin; hatun-yuyay.

\section{INTRODUCCIÓN}

En granjas de reproductoras, la eficiencia de las mismas se evalúa por la productividad numérica. Los factores técnicos que se estudia para el análisis de la productividad de las explotaciones porcinas dependen de los intereses de cada país o de cada región. (Marco y Collell, 2009), agrupan los parámetros para medir la eficiencia reproductiva en cuatro grupos: Estructura de la explotación; Ritmo de partos; Prolificidad y Lactación.

En la actualidad, la producción porcina se encuentra con la problemática de que existen muchos estudios que tratan el tema de la productividad numérica de las cerdas; pero estos estudios se realizan en condiciones de manejo muy diferentes. Una técnica que permite resolver este problema desde el punto de vista estadístico es el meta-análisis, en el cual las conclusiones individuales de diferentes estudios, con una misma finalidad, son integradas y analizadas a través de un proceso estadístico y se puede proporcionar una estimación más precisa del efecto de cada factor reproductivo en la productividad numérica de las cerdas, así como explicar la variabilidad de los efectos entre factores, según los estudios presentes en la bibliografía. En la literatura se ha empleado diferentes métodos para la realización de meta-análisis en múltiples estudios realizados en la producción animal y específicamente en la producción porcina, (Chizzotti, Tedeschi y Valadares, 2008; Sales y Jancík, 2011; Schulin-Zeuthen et al. 2007).

\section{MATERIALES Y MÉTODOS}

Para el presente estudio se seleccionaron referencias bibliográficas de todas las encontradas sobre el tema en el ámbito literario; para ello se establecieron como criterios de inclusión que: (1) las investigaciones tenían que ser desarrolladas en condiciones de producciones reales; (2) la influencia o no de los factores tenía que quedar dem- 
ostrada estadísticamente y tenían que aportar datos como el tamaño de la muestra, la media, desviación estándar, el coeficiente de correlación o los valores de probabilidad obtenidos por un análisis de varianza por software conocidos como SPSS, MINITAB, Statgraphics o InfoStat; (3) los estudios tenían que estar publicados en revistas de impacto internacional o en eventos reconocidos internacionalmente y (4) aunque los autores refiriesen otros factores, para agruparlos se escogería aquel factor que mayor importancia tenía dentro de la investigación publicada.

Identificación de la literatura y metodología para la búsqueda bibliográfica. La búsqueda bibliográfica se realizó en cuatro fases:

Fase 1. Búsqueda informatizada en las bases de datos del Bdporc, PigCHAMP; Java Specification Request e Intervet.

Fase 2. Búsqueda en las revistas reconocidas internacionalmente y que tienen un factor de impacto en Europa y América: Journal of Animal Science; Animal Practice; Journal of Reproductive Fertility; Animal Production; Livestock Production Science; Revista Electrónica de Veterinaria; Navarra Agraria; Mundo Ganadero; Deseases of Swine; Swine Production Management; Large Animal Veterinarian; Livestock Research for Rural Development; Journal American Veterinary Society; Ciencia y Cultura Latinoamericana; American Association of Swine Practitioners; Animal Research; Revista Computadorizada de Producción Porcina; British Journal of Nutrition; Journal of Animal Physiology and Animal Nutrition; Zootecnia Tropical; British Society of Animal Production; Veterinary Clinics of North America; Journal Nutrition; Journal Animal Physiology Animal Nutrition; Theriogenology, ANAPORC y Animal Physiology and Animal Nutrition.

Fase 3. Se revisaron las publicaciones procedentes de universidades de reconocimiento internacional como University of Iowa Press Ames; University of New England; University of Minnesota; Nottingham University Press; National Academy Press, Washington D.C y la Universidad Michoacana de «San Nicolás de Hidalgo».

Fase 4. Se recopiló artículos publicados en eventos internacionales que incluyeron el XXXI Congreso Nacional de la Asociación de Médicos Veterinarios Especialistas en Cerdos, México; 27th Annual Meeting of the American Association of Swine Practitioners; I Curso Internacional de Reproducción Porcina; Academia de Investigación en Biología de la Reproducción, México; XIII, XXI, XIII y X Curso de especialización FEDNA; Corporación Ganadera (CORFOGA) y 12th International Pig Veterinary Society (IPVS) Congress.

Se escogieron 96 bibliografías en idioma español, inglés y portugués que constituyeron el universo; de donde solo se tomaron las citas. Las variables de clasificación fueron los factores que influyen en la productividad numérica de las cerdas, el agrupamiento se muestra en la tabla 1. 


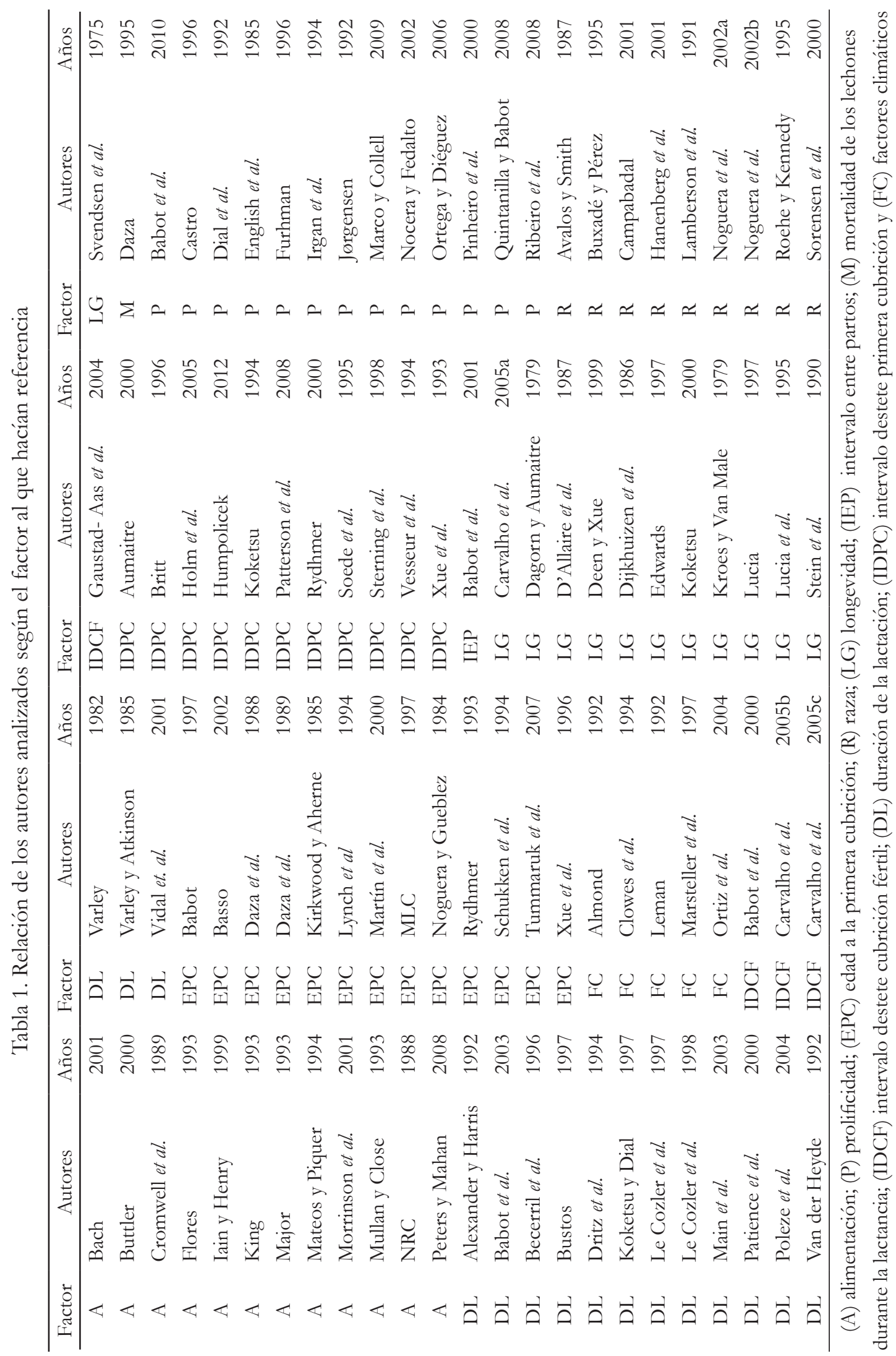


Las variables categóricas refieren el nivel de influencia de estos factores en la productividad numérica y se codificaron según el grado de significación referido en la investigación como factor analizado que influye o no y si influye es de forma directamente proporcional (representado en los datos por el signo $(+)$ o inversamente proporcional, representado en los datos por el signo (-). Para cada factor se estableció una de las clasificaciones que se observan en la tabla 2. La identificación de los factores está representada en el Dendograma A y la agrupación de los mismos según similitud por el Dendograma B, de la figura 1 .

Para codificar las variables categóricas se siguieron seis pasos consistentes en (1) limpieza de los textos; (2) reducción de las palabras a su raíz; (3) creación de una matriz; (4) determinación de los pesos de cada palabra en cada opinión; (5) representación de cada opinión por un vector de palabras y (6) agrupamiento de opiniones en base a los vectores de palabras. Para analizar estadísticamente los datos obtenidos, se realizó una prueba de corridas con el objetivo de determinar la existencia de un sesgo gradual en la selección de las citas o si no se han seleccionado al azar y tras confirmar la aleatoriedad se efectuó un análisis de conglomerado jerárquico para determinar las similitudes de los conglomerados de las observaciones y de las variables categóricas, la similitud, S(ij), entre dos conglomerados i y j está dada por:

$$
\mathrm{S}(\mathrm{ij})=100(1-\mathrm{d}(\mathrm{ij}) / \mathrm{d}(\mathrm{máx})
$$

Donde: d(máx) es el valor máximo en la matriz de distancia original, D.

Tabla 2. Codificación de la influencia de los factores en la productividad numérica

\begin{tabular}{ccccc}
\hline $\begin{array}{c}\text { Codificación de } \\
\text { cada factor }\end{array}$ & $\begin{array}{c}\text { Influencia } \\
\text { positiva }\end{array}$ & $\begin{array}{c}\text { Color } \\
\text { asignado }\end{array}$ & $\begin{array}{c}\text { Influencia } \\
\text { negativa }\end{array}$ & $\begin{array}{c}\text { Color } \\
\text { asignado }\end{array}$ \\
\hline Influencia alta & +1 & rojo & -1 & amarrillo \\
Influencia media & $+0,5$ & rosado & $-0,5$ & malva \\
Influencia baja & $+0,25$ & verde & $-0,25$ & marrón \\
No influye & 0 & azul & 0 & azul \\
\hline
\end{tabular}

Finalmente, se realizaron dos análisis de varianza para encontrar diferencias entre las medias de los conglomerados, uno primero para los factores que tienen una relación directamente proporcional con la productividad numérica y otro para los factores que tenían una relación inversamente proporcional. Para ello se empleó el programa MINITAB.Verción 16 en español. 


\section{RESULTADOS}

La tabla 3 presenta los porcentajes de aparición de las citas por años y factores; las investigaciones más recientes se centran en los intervalos entre partos, la longevidad y el intrevalo destete cubrición fértil.

Tabla 3. Porcentajes de aparición de las citas relacionadas con la productividad numérica

\begin{tabular}{|c|c|c|c|c|c|c|c|c|c|c|c|c|}
\hline & \multicolumn{12}{|c|}{ Factores } \\
\hline Años & UM & A & $\mathrm{P}$ & EPC & $\mathrm{R}$ & LG & IEP & M & IDCF & DL & IDPC & $\mathrm{FC}$ \\
\hline $\begin{array}{c}\text { Antes de } \\
1990\end{array}$ & $(\%)$ & 18,2 & 13,3 & 0,0 & 30,0 & 0,0 & 0,0 & 100,0 & 20,0 & 21,9 & 17,6 & 27,3 \\
\hline 1990-1994 & $(\%)$ & 30,3 & 40,0 & 20,0 & 0,0 & 0,0 & 0,0 & 0,0 & 0,0 & 24,5 & 41,2 & 18,1 \\
\hline 1995-1999 & $(\%)$ & 15,1 & 6,7 & 40,0 & 60,0 & 25,0 & 0,0 & 0,0 & 0,0 & 19,5 & 17,6 & 27,3 \\
\hline $2000-2004$ & $(\%)$ & 18,2 & 20,0 & 40,0 & 10,0 & 0,0 & 0,0 & 0,0 & 20,0 & 21,9 & 23,6 & 27,3 \\
\hline $\begin{array}{l}\text { Después } \\
\text { de } 2004\end{array}$ & $(\%)$ & 18,2 & 20,0 & 0,0 & 0,0 & 75,0 & 100,0 & 0,0 & 60,0 & 12,2 & 0,0 & 0,0 \\
\hline
\end{tabular}

La matriz que recoge la clasificación de las citas, según el factor al que hacen referencia, fue sometida a una prueba de corridas y los resultados mostraron que los valores de $p$ resultantes para cada factor son mayores al nivel de significación $(\alpha=0,05)$, por lo que existe suficiente evidencia para concluir que los datos están en orden aleatorio (tabla 4).

Tabla 4. Valores de probabilidad obtenidos de la Prueba de corridas

\begin{tabular}{cccc}
\hline Factores & $\mathrm{p}(\boldsymbol{\alpha}=0,05)$ & Factores & $\mathrm{p}(\boldsymbol{\alpha}=0,05)$ \\
\hline A & 0,637 & $\mathrm{M}$ & 0,881 \\
$\mathrm{P}$ & 0,062 & IDCF & 0,56 \\
EPC & 0,124 & DL & 0,213 \\
R & 0,914 & IDPC & 0,355 \\
LG & 0,359 & FC & 0,18 \\
IEP & 0,881 & & \\
\hline
\end{tabular}


La figura muestra que la mayoría de las investigaciones centran sus estudios en la importancia de la prolificidad, longevidad, mortalidad y duración de la lactación (figura 1)

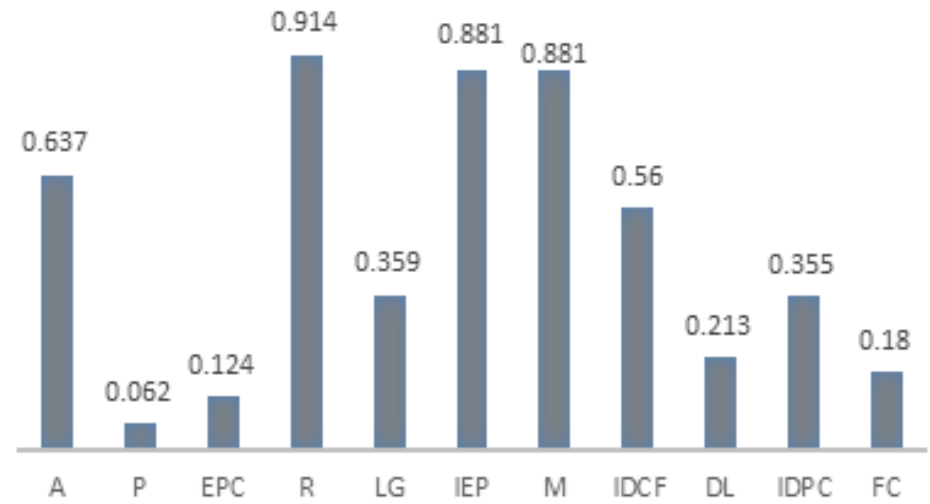

Figura 1. Valores de probabilidad para determinar la aleatoriedad de las citas bibliográficas, para $\alpha=0,05$

La figura 2(A) muestra el Dendograma para las observaciones. En este caso agrupa las citas bibliográficas de los autores que realizan planteamientos similares sobre la influencia de cada variable en los lechones destetados por cerda y año; el primer conglomerado de variables muestra una similitud grande entre ellas, a partir de lo cual se forman otros conglomerados que agrupan el resto de las variables, como se observa en el conglomerado de color azul, que corresponde a las observaciones, en la matriz, que no influyen; es el más grande y en él están agrupados la mayor cantidad de autores. Obsérvese que este resultado está relacionado con la matriz creada con todas las posibles codificaciones por autor en cada factor; así el menor conglomerado es el que corresponde a las citas que plantean una influencia baja positiva, lo que se explica dada la selección de las bibliografías en cuanto a su rigor científico y las fuentes consultadas.

La figura 2(B) muestra la agrupación en conglomerados de las variables y se puede distinguir perfectamente dos grupos de variables: grupo 1, los que según la literatura consultada influyen de forma positiva en una u otra medida en la productividad numérica (encerrado en rectángulo azul); grupo 2, los que influyen negativamente, (encerrados en rectángulo negro) a partir de este grupo de variables se va formando subgrupos y dentro de ellos se agrupan las variables por similitud entre ellas y el grado de influencia sobre la productividad numérica. La interrelación entre ambos grupos está definida por el factor Longevidad. 


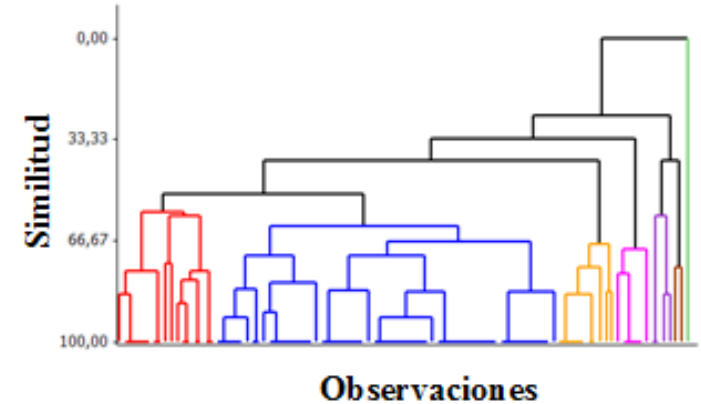

A

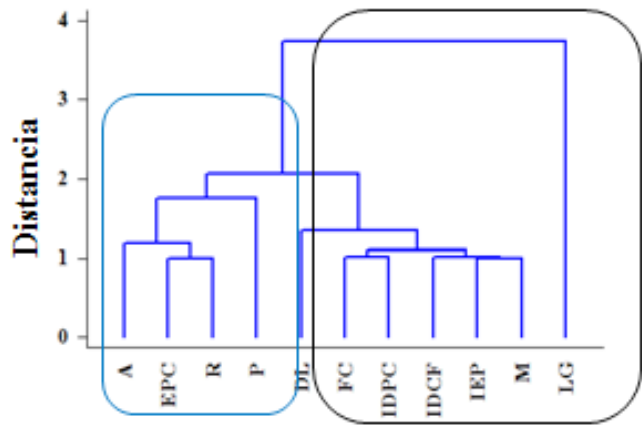

B

Figura 2. Dendogramas de las observaciones (A) y las variables (B) que influyen en la productividad numérica de las cerdas

El análisis de varianza en ambos casos tuvo valores de $p$ inferiores al de $\boldsymbol{\alpha}=\mathbf{0 , 0 5}$, por lo que existen diferencias significativas entre la influencia de los factores en la productividad numérica de las cerdas. En la tabla 5 se observa el orden de influencia, la A y P tienen mayor influencia de modo directamente proporcional; mientras que los restantes tienen una influencia inversa, siendo menos significativo en la DL y sin diferencia alguna entre M, IEP, IDCF, FC y IDPC; entonces se interpreta que las cerdas con mejor alimentación, mejores medias de prolificidad, razas más especializadas y una mayor edad a la primera cubrición incrementarán su productividad numérica; sin embargo, a mayor mortalidad, intervalo entre partos, intervalo destete cubrición fértil, intervalo destete primera cubrición, mayor temperatura ambiental, cerdas muy longevas o con duraciones de lactación muy largas, disminuirán su productividad numérica.

Tabla 5. Análisis de varianza para los conglomerados

\begin{tabular}{cccccc}
\hline Factores & Media & $\begin{array}{c}\text { Agrupación } \\
\text { (Fisher) }\end{array}$ & Factores & Media & $\begin{array}{c}\text { Agrupación } \\
\text { (Fisher) }\end{array}$ \\
\hline A & 0,323 & A & M & $-0,010$ & A \\
P & 0,197 & B & IEP & $-0,010$ & A \\
R & 0,061 & C & IDCF & $-0,025$ & A \\
EPC & 0,035 & C & FC & $-0,051$ & A \\
& & & IDPC & $-0,056$ & A \\
& & & LG & $-0,061$ & A \\
& & & DL & $-0,384$ & B \\
\hline
\end{tabular}

A-B-C, letras diferentes reflejan diferencias significativas para $\mathrm{p}$-valor $\leq 0,05$ 
Aunque los resultados matemáticos sitúen la DL a mayor distancia de la M, IEP, IDCF, FC, IDPC y LG; también es importante analizar que la DL varía muy poco, de 21 a 28 días, lo que hace que su influencia en la productividad numérica esté más ligada al efecto sobre la prolificidad de la siguiente camada que a los lechones destetados por cerda y año.

\section{DISCUSIÓN}

Los diferentes métodos para la realización de meta-análisis empleados en la producción porcina, han tenido como objetivo relacionar índices productivos según estudios realizados previamente (Chizzotti, Tedeschi y Valadares, 2008; Sales y Jancík, 2011; Schulin-Zeuthen et al., 2007). En este trabajo se ha creado una metodología que recoge técnicas descritas en estas referencias, en todos los casos los meta-análisis tienen como fin establecer una relación entre el aspecto a estudiar y los estudios existentes sobre el tema. Las publicaciones revisadas analizan la productividad por cerda y año como un factor que depende de un grupo de factores más que lo modifican en el manejo diario de la granja, los cuales pueden estar influenciados por el hombre o no, en dependencia de la relevancia de los mismos.

Al prever una productividad numérica para una granja en cuestión se debe tener muchos elementos en cuenta que no siempre pueden ser controlados (Nocera y Fedalto, 2002 y Carvalho et al., 2005). Todos los factores que intervienen en la productividad numérica de las cerdas no se pueden controlar al unísono, por ello la mayoría de los investigadores centran sus estudios en aquellos que más relevancia tienen desde el punto de vista de su influencia (English, Smith y Mclew, 1985; Dial, Marsh y Polson, 1992; Castro, 1996; Furhman, 1996 y Daza, 1995), lo cual está reflejado en los resultados de este trabajo.

Los resultados del analisis de varaianza coinciden con los estudios realizados por Bach (2001); Babot et al. (2010); Campabadal (2001) y Martín et al. (2000), respectivamente.

Sin embargo, a mayor mortalidad, intervalo entre partos, intervalo destete cubrición fértil, intervalo destete primera cubrición, mayor temperatura ambiental, cerdas muy longevas o con duraciones de lactación muy largas, disminuirán su productividad numérica. Estos resultados se corresponden con Daza (1995); Babot, Vidal y Chávez (2001); Carvalho, Sousa y Bastos (2005), Aumaitre (2000); Ortíz, Ortega y Becerril (2004); Quintanilla y Babot (2008) y Babot et al. (2003).

Aunque los resultados matemáticos sitúen la DL a mayor distancia de la M, IEP, IDCF, FC, IDPC y LG, también es importante analizar que la DL varía muy poco, de 21 a 28 días, lo que hace que su influencia en la productividad numérica esté más ligada al efecto sobre la prolificidad de la siguiente camada que a los lechones destetados por cerda y año, el efecto significativo de la DL sobre la siguiente camada fue descrito por Poleze et al, (2004). 
Todos los factores se interrelacionan entre ellos (Babot, Vidal y Chávez, 2001) por lo que un análisis individualizado resulta muy difícil; sin embargo el meta-análisis nos permite conocer las relaciones que los definen y el orden de prioridad que las investigaciones les proporcionan, (Schulin-Zeuthen et al., 2007).

Todos los factores se interrelacionan entre ellos (Babot, Vidal y Chávez, 2001) por lo que un análisis individualizado resulta muy difícil; sin embargo el meta-análisis nos permite conocer las relaciones que les definen y el orden de prioridad que las investigaciones les proporcionan, (Schulin-Zeuthen et al., 2007).

\section{CONCLUSIONES}

La agrupación de los resultados de investigación más relevantes a través del meta-análisis permitió determinar el grado y sentido de la influencia que tienen los factores que, a juicio de los autores, modifican la productividad numérica de las cerdas; donde se reconoce que los estudios principales van encaminados a determinar la edad o ciclos que una cerda debe permanecer en un rebaño, tomando en consideración la prolificidad por orden de parto, así como acortar los intervalos entre partos y destete cubrición fértil y en qué porcentaje la raza, la edad a la primera cubrición y la duración de la lactación son los que menos nivel de influencia tienen.

\section{AGRADECIMIENTOS}

Este trabajo ha sido parcialmente apoyado por la Red CYTED 516RT0513 «Red Iberoamericana de Agro-bigdata y «Decision Support Systems» (DSS) para un sector agropecuario sostenible».

\section{REFERENCIAS BIBLIOGRÁFICAS}

Aumaitre, Aimé. 2000. «Sistema de manejo de alta productividad para cerdas en Europa», ANAPORC, 203. 55-86.

Babot, Daniel. y otros. 2010. «Gestión técnica y económica de explotaciones porcinas: España y Catalunya en el contexto de Europa». Memorias del X Congreso Nacional de Producción Porcina, Mendoza, Argentina. <http://www.produccion-animal.com.ar/ produccion_porcina/00-X_congreso/11-gestion.pdf $>$ [Consulta: 21-05-2016].

Babot, Daniel.; Vidal, José y Chávez, Eduardo. 2001. Decisiones de manejo en granjas y sistemas de producción alternativos. En Babot, D. (Ed.) Gestión en empresas de producción porcina. Análisis, diagnóstico y toma de decisiones. Universitat de Lleida. 101.140. ISBN: 84-8409-108-2. 
Babot, Daniel. y otros. 2003. «Evolución comparada de la productividad de las explotaciones porcinas en España, Francia y Holanda (1990-2000)». ANAPORC, 230. 68-83.

Bach, Alex. 2001. «La reproducción del vacuno lechero: Nutrición y Fisiología». XVII Curso de Especialización. Avances en nutrición y alimentación animal. FEDNA. España.

Campabadal, Carlos y Navarro, Héctor. 2001. «Alimentación de los cerdos en condiciones tropicales». Asociación Americana de Soya. México. 279.

Carvalho, Luiz; Sousa Karine y Bastos, Francisco. 2005. «Efeito do intervalo desmame cio sobre a prolificidade da matriz suína». En: XII Congresso Brasileiro de Veterinários Especialistas em Suinos, Fortaleza: Abraves, 2205. v . 1. 212-213.

Carvalho, Luiz y otros. 2005. «Efeito da ordem de parto sobre a prolificidade da fêmea suína». En: XII Congresso Brasileiro de Veterinários Especialistas em Suinos. Fortaleza: Abraves, 2005. v . 1. 214-215.

Castro, G. 1996. «Factors to benchmark for maximum efficiency». 27th Annual Meeting of the American Association of Swine Practitioners. 317-321.

Chizzotti, Mario; Tedeschi Luis and Valadares, Filho. 2008. «A meta-analysis of energy and protein requirements for maintenance and growth of Nellore cattle». Journal Animal Science. 86:1588-1597.

Daza, Andrada. 1995. «Explotaciones de ganado reproductor (I). Estrategias y gestión-técnico económica». Mundo Ganadero. N.9. 48-56 .

Dial, Gary; Marsh, Willian y Polson, Dale. 1992. «Reproductive failure: differential diagnosis. Diseases of Swine». University of Iowa Press. Ames. 88-137.

English, Peter; Smith, William y Mclew, Alastair. 1985. «La cerda: cómo mejorar su productividad». Manual Moderno. México D.F. 196-233.

Fuhrman, Monte. 1996. «Hog heaven? Pig production techniques in México». Large Animal Veterinarian, 14:26-30.

Marco Enric y Collell, Miquel. 2009. «Análisis y diagnóstico de problemas reproductivos». < <http://www.masterporcino.org $>$ [Consulta: 23-07-16].

Martín, Santiago y otros. 2000. «Efecto del aparato genital de la primeriza sobre la productividad de la cerda». ANAPORC. 198. 63.

Nocera, Paulo y Fedalto, Luis. 2002. «A influência de fatores ambientais e de inseminação artificial sobre as características produtivas de suínos». Archives of Veterinay Science, 7. 159-172.

Ortíz, Ruy; Ortega,Victor y Becerril, José. 2004. «Efectos ambientales en cerdas sometidas a lactancias de 12 y 21 días en México. Rasgos del comportamiento reproductivo». Revista Computadorizada de Producción Porcina. 3. 
Poleze, Evandro y otros. 2004. «Conseqüências reprodutivas da variação do intervalo- desmama -estro em suínos». En: Anais do XI Congresso Brasileiro de Veterinários Especialistas em Suinos. Goiânia. Anais. Fortaleza, Ceará. 187-188.

Quintanilla, Raquel y Babot, Daniel. 2008. Gestión de la producción y de la empresa porcina: parámetros implicados en el rendimiento técnico de las explotaciones porcinas. En: Babot, D. (Ed.), La gestión técnica de explotaciones porcinas en España. Ed. Ministerio de Agricultura, Pesca y Alimentación.

Sales, José and Jancík, F. 2011. «Effects of dietary chromium supplementation on performance, carcass characteristics, and meat quality of growing-finishing swine: A meta-analysis». Journal Animal Science. 4054-4067.

Schulin-Zeuthen, Mette. 2007. «Meta-analysis of phosphorus balance data from growing pigs». Journal Animal Science. 1953-1961.

Fecha de recepción: 10 de junio de 2016

Fecha de aceptación: 14 de noviembre de 2016

\section{Correspondencia}

Yulien Fernández Romay

brianaamalia2003@gmail.com 